\title{
Online Monitoring Scheme Using PCA through Kullback-Leibler Divergence Analysis Technique for Fault Detection
}

\author{
Wahiba Bounoua ${ }^{1, *}$, Amina B. Benkara ${ }^{1}$, Abdelmalek Kouadri ${ }^{1}$, Azzeddine Bakdi ${ }^{2}$ \\ wb.bounoua@gmail.com (Corresponding author),aminabenkara26@gmail.com, ab_kouadri@hotmail.com,bkdaznsun@gmail.com
}

1. Signals and Systems Laboratory, Institute of Electrical and Electronics Engineering, University M'Hamed Bougara of Boumerdes, Avenue of independence, 35000 Boumerdès, Algeria

2. Department of Mathematics, University of Oslo, 0851 Oslo, Norway

\begin{abstract}
Principal Component Analysis (PCA) is a common tool in the literature and widely used for process monitoring and fault detection. Traditional PCA is associated with the two well-known control charts, the Hotelling's $\mathrm{T}^{2}$ and the Squared Prediction Error (SPE), as monitoring statistics. This paper develops the use of new measures based on a distribution dissimilarity technique named Kullback-Leibler Divergence (KLD) through PCA by measuring the difference between online estimated and offline reference density functions. For processes with PCA scores following a multivariate Gaussian distribution, KLD is computed on both principal and residual subspaces defined by PCA in a moving window to extract the local disparity information. The potentials of the proposed algorithm are afterwards demonstrated through an application on two well-known processes in chemical industries; the Tennessee Eastman process as a reference benchmark and three tank system as an experimental validation. The monitoring performance was compared to recent results from other Multivariate Statistical Process Monitoring (MSPM) techniques. The proposed method showed superior robustness and effectiveness recording the lowest average missed detection rate and false alarm rates in process fault detection.
\end{abstract}

Key words: Kullback-Leibler divergence; principal component analysis; Tennessee Eastman process; fault detection; three tank system. 


\section{Introduction}

Process monitoring is becoming a major concern in modern industries, and particularly in chemical processes. This is due to the increasing system complexity and the need for improving productivity and saving machines from serious damages. For this sake, much research is being conducted continuously for developing new tools that enhance process Fault Detection and Diagnosis (FDD). These tools include the model-based approaches such as (Chen \& Patton, 1999) and (Ding, 2008) relying heavily on a priori knowledge about the mathematical and physical relationships of the system. The most common approaches include filters (Frank, et al., 2000; Zhuang, et al., 2014; Zhuang, et al., 2016), observers and parameter estimations (Alcorta García \& Frank, 1997; Wang, 2012). These methods might be intricate because it is still difficult in numerous cases to set up the most adequate model and cannot be scaled to large-scale systems. Alternatively, more effective techniques recently emerged for complex processes are based on Multivariate Statistical Process Monitoring (MSPM) strategies that learn from huge historical data to build the FDD framework (Hauck, et al., 1999; Xiong, et al., 2007). The main advantages of these techniques are the ability to reduce the dimensionality of the data and deal with highly correlated variables to extract the most informative features, in addition to the flexibility in their derivation and implementation. Principal Component Analysis (PCA) (Jackson, 1991), the most commonly used MSPM method, projects the original data into a lower dimensional space while tracking the maximum variance and hence splitting the original space into an approximated principal subspace and a residual subspace. For fault detection PCA conventionally incorporates two control charts for the analysis, the Hotelling's $\mathrm{T}^{2}$ and the Squared Prediction Error (SPE), calculated based on an off-line PCA reference model for each new observation in on-line monitoring (Guo, et al., 2018; Venkatasubramanian, et al., 2013; Wade, et al., 2005). The Hotelling's $T^{2}$ represents the approximate model that indicates how far each sample is from the centre of the model. On the other hand, SPE contributes to the lack-of-fit of the approximate model to the one it was originally brought from. As a matter of fact, diverse extensions have been introduced to PCA in order to overcome some of its limitations such as Nonlinear PCA (NLPCA) (Jia, et al., 1998), considering the system's 
dynamics using Dynamic PCA (DPCA) (Ku, et al., 1995), Kernel PCA (KPCA) (Lee, et al., 2004; Zhongda, et al., 2016), and Multiscale PCA (MSPCA) (Mirin, 2013) that combines the PCA with wavelet analysis. However, the control charts still need more thoughtful attention from researchers in order to find new alternatives that further enhance the process monitoring efficiency. As stated by Wang \& Chen (2004), an increase in $\mathrm{T}^{2}$ may not always be due to real faults, it might be just a shift in the operating region because changes in $\mathrm{T}^{2}$ are consistent with the model. This gives rise to more false alarms under similar situations and makes the SPE a better fault indicator. However, it has been shown experimentally on real data that the Qstatistic is more sensitive to the modelling errors (Harkat, et al., 2000). With such paradox, our intent is to provide a new approach for the monitoring statistics to achieve reliable monitoring rather than changing the data transformation technique.

In the current paper, the Kullback-Leibler Divergence (KLD) is to be investigated as a control chart for fault indication. This divergence measure was heavily used recently and applied successfully in many different ways for process monitoring purposes. Aggoune, et al. (2016) examined Kullback-Leibler divergence in detecting abnormal modes after developing a NARMAX polynomial model to estimate the nonlinear behavior of the plant. In another study (Harmouche, et al., 2014), KLD was used in univariate analysis of individual principal scores obtained from PCA model of the data, where KLD showed better results over the $\mathrm{T}^{2}$ statistic in detecting incipient faults. This study was pursued by the fault diagnosis phase and detecting incipient faults in a noisy environment (Harmouche, et al., 2015). The same logic was followed by Youssef, et al. (2016) with an optimal threshold established depending on the signal to noise ratio and the fault to noise ratio. In these studies, KLD was computed over single scores of the principal subspace only; therefore, they provide a number of fault detectors equivalent to the number of principal components while completely ignoring the residual subspace and losing the key advantage of dimension reduction of PCA. This could be a key problem for plant-wide processes where the number of variables is considerably large, besides neglecting the residual subspace that holds worthy information of the process state (Gertlert, et al., 1999). To this end, we propose a new approach 
for fault detection following the fact that when constructing an accurate PCA model, the changes in process measurements will appear primarily as changes in the PCA scores. Thereafter, two monitoring statistics based on KLD are used to measure the distance between the multivariate scores distributions in healthy and faulty states of the principal as well as the residual subspaces and their sensitivity is tested to the different types of faults. As a result, the overall monitoring of a large-scale process is reduced to controlling the two developed statistics which appear very potentially effective and complementing one another performance as demonstrated on a large realistic benchmark and an experimental application.

This work is commenced by describing the different notions and definitions of the utilized techniques in Section 2. Section 3 is dedicated to explaining the proposed methodology. Section 4 summarizes the application procedure. Simulation and discussions of the results on the TEP and three tank system are presented in Section 5 and Section 6, respectively. And finally, section 7 draws some concluding remarks and lists some hints for future work.

\section{Preliminaries}

\subsection{PCA-based modelling}

Considering the acquired data in the form of a matrix $\boldsymbol{X}_{0}$, collected from a process in normal operation with $N$ samples of $m$ variables, the data matrix $\boldsymbol{X}_{0}$ is first normalized to zero mean and unit variance giving the normalized matrix $\boldsymbol{X}$ :

$$
\boldsymbol{X}=\left[\begin{array}{llll}
\boldsymbol{x}_{1} & \boldsymbol{x}_{2} & \cdots & \boldsymbol{x}_{m}
\end{array}\right] \in \mathcal{R}^{N \times m}
$$

PCA transforms this data matrix into a new matrix $T \in \mathcal{R}^{N \times m}$ named the score matrix of uncorrelated variables $\boldsymbol{t}_{1}, \boldsymbol{t}_{2}, \cdots, \boldsymbol{t}_{m}$ :

$$
T=X P
$$

$\boldsymbol{P}$ is the loading matrix with orthogonal components i.e. $\boldsymbol{P} \boldsymbol{P}^{T}=\boldsymbol{I}$ constructed by the eigenvectors that represent the variation directions, it is obtained by an orthogonal transformation of the covariance matrix $\boldsymbol{\Phi}$ through the 
Singular Value Decomposition (SVD). The SVD is a generalization of the eigen-decomposition and orthogonal decomposition when dealing with symmetric matrices. Performing the SVD on the sample covariance matrix is given as:

$$
\boldsymbol{\Phi}=\frac{1}{N-1} \boldsymbol{X}^{T} \boldsymbol{X}=\boldsymbol{P} \boldsymbol{\Lambda} \boldsymbol{P}^{T}
$$

where $\Lambda=\operatorname{diag}\left(\lambda_{1}, \lambda_{2}, \cdots, \lambda_{m}\right)$ is the diagonal eigenvalue matrix, in a decreasing order representing the amount of variance $\lambda_{1} \geq \lambda_{2} \geq \cdots \geq \lambda_{m} \geq 0$.

Data dimensionality reduction can be achieved by splitting $\boldsymbol{P}$ and $\boldsymbol{\Lambda}$ into modeled and non-modeled variations, the first part $\boldsymbol{P}_{p c} \in \mathcal{R}^{m \times l}$ and $\boldsymbol{\Lambda}_{p c} \in \mathcal{R}^{l \times l}$ spanning the principal subspace and the other part is $\boldsymbol{P}_{\text {res }} \in \mathcal{R}^{m \times(m-l)}$ and $\boldsymbol{\Lambda}_{\text {res }} \in \mathcal{R}^{(m-l) \times(m-l)}$ spanning the residual subspace:

$$
\boldsymbol{\Lambda}=\left[\begin{array}{cc}
\boldsymbol{\Lambda}_{p c} & 0 \\
0 & \boldsymbol{\Lambda}_{r e s}
\end{array}\right], \quad \boldsymbol{P}=\left[\begin{array}{ll}
\boldsymbol{P}_{p c} & \boldsymbol{P}_{r e s}
\end{array}\right]
$$

Hence, the score matrix $\mathbf{T}$ can be rewritten as:

$$
\boldsymbol{T}=\boldsymbol{X P}=\boldsymbol{X}\left[\begin{array}{ll}
\boldsymbol{P}_{p c} & \boldsymbol{P}_{r e s}
\end{array}\right]
$$

giving,

$$
\widehat{\boldsymbol{T}}=\boldsymbol{X} \boldsymbol{P}_{p c} \quad \text { and } \quad \widehat{\boldsymbol{X}}=\widehat{\boldsymbol{T}} \boldsymbol{P}_{p c}{ }^{T}
$$

$\widehat{\boldsymbol{T}}$ represents the projection of $\boldsymbol{X}$ on the first $l$ largest eigenvectors of the sample covariance matrix. And $\widetilde{\boldsymbol{T}}$ is the projection of $\boldsymbol{X}$ on the last $m-l$ eigenvectors.

$$
\widetilde{\boldsymbol{T}}=\boldsymbol{X} \boldsymbol{P}_{\text {res }} \quad \text { and } \quad \widetilde{\boldsymbol{X}}=\widetilde{\boldsymbol{T}} \boldsymbol{P}_{\text {res }}{ }^{T}
$$

where $\widetilde{\boldsymbol{X}}$ represents the matrix of residuals denoted by $\boldsymbol{E}$. As a result, PCA decomposes the original data set $\boldsymbol{X}$ into:

$$
\boldsymbol{X}=\widehat{\boldsymbol{X}}+\boldsymbol{E}
$$




\subsection{PCA model selection}

Various methods have been suggested for determining the appropriate number of principal components that split up the informative subspace and the residual one (Valle, et al., 1999). Most of these methods are subjective because they use a monotonically decreasing index for analysis. The Variance of Reconstructed Error (VRE) and the Cumulative Percentage of Variance (CPV) techniques are chosen in our work and applied on the faultfree dataset to select the adequate number of components to keep for constituting the PCA-model. Unlike most other methods reported in the literature, the VRE method has a guaranteed minimum over the number of scores corresponding to the best reconstruction. Therefore, it avoids the arbitrariness of other methods with monotonic indices (Qin \& Dunia, 2000). In addition to that, the CPV criterion is chosen as being the most used standard method that allows selecting the number of scores for a predetermined variance percentage.

\subsubsection{Variance of Reconstruction Error}

The Variance of the Reconstruction Error (VRE) (Dunia \& Qin, 1998) selects the number of principal components based on the best reconstruction of the process variables. VRE index has an important characteristic as it reaches a minimum value corresponding to best reconstruction. When the PCA model is used for faulty sensors reconstruction, the VRE would be a function of the number of Principal Components (PCs). The minimum then found through VRE calculation determines the optimal number of PCs. This criterion utilizes certain notions brought up by Dunia, et al (1996) that define the principle of reconstruction.

The principle of reconstruction consists of estimating the $i$ th variable from the remaining variables in measurement vector $\boldsymbol{X} \in \mathcal{R}^{N \times m}$. Dunia, et al. (1996) consider that the sensor measurement is corrupted with a fault along a direction $\xi_{i} \in \mathcal{R}^{m}$ of magnitude $h$, thus, the actual sensor measurement is

$$
\boldsymbol{x}=\boldsymbol{x}^{*}+h \xi_{i}
$$


with $\boldsymbol{x}^{*}$ denotes the normal sample vector and $\left\|\xi_{i}\right\|=1$ corresponds to the $i$ th column of an identity matrix. The aim is to find an estimate for $\boldsymbol{x}^{*}$ along the direction of the fault $\boldsymbol{\xi}_{i}$ that best correct the effect of the fault. Strictly speaking, we try to find $h_{i}$ such that

$$
\boldsymbol{x}_{i}^{r e c}=\boldsymbol{x}-h_{i} \xi_{i}
$$

is most consistent with the PCA model or has a minimum model error in the sense of the least-squares method. The variance of reconstruction error is then defined by Qin \& Dunia (2000) as:

$$
u_{i}(l)=\operatorname{var}\left(\xi_{i}^{T} \boldsymbol{x}-\boldsymbol{x}_{i}^{r e c}\right)
$$

The task of finding the number of PCs is to minimize $u_{i}$ with respect to $l$. Considering all data dimensions, the VRE to be minimized is defined as:

$$
\operatorname{VRE}(l)=\sum_{i=1}^{m} \frac{u_{i}(l)}{\operatorname{var}\left(\xi_{i}^{T} \boldsymbol{x}\right)}=\sum_{i=1}^{m} \frac{u_{i}(l)}{\boldsymbol{\xi}_{i}^{T} \boldsymbol{R} \xi_{i}}
$$

$\boldsymbol{R}$ is the correlation matrix and $l$ is the number of retained PCs.

Although this criterion was derived for fault reconstruction, it is applicable for other purposes, just by using normal data only without requiring actual faults to occur.

\subsubsection{Cumulative Percent of Variance}

CPV (Malinowski, 1991) is a straightforward criterion for estimating the number of nontrivial components by including all components up to a predetermined percentage of the total variance. The CPV captured by the first $l$ PCs is calculated by (Xia, et al., 2013):

$$
\operatorname{CPV}(l)=100\left(\frac{\sum_{j=1}^{l} \lambda_{j}}{\sum_{j=1}^{m} \lambda_{j}}\right) \%
$$

Hence choosing those $l$ PCs turns out to a matter of choosing the percentage of total variance we desire to keep. 


\subsection{Kullback-Leibler Divergence}

\subsubsection{Definition}

Kullback-Leibler (KL) divergence, also called relative entropy, information divergence or I-divergence, is a special case of the f-divergence family for dissimilarity computations that was introduced by Kullback \& Leibler (1951). KL divergence is an information-based measure of dissimilarity between two probability distributions $f_{Y}$ and $\tilde{f}_{Y}$ defined over the same random variable $Y$. Typically, $f_{Y}$ represents the true distribution and $\tilde{f}_{Y}$ represents a model or approximation of $f_{Y}$ (Wang, et al., 2014). In other words, KL divergence can be interpreted as a measure of the inefficiency of assuming that the distribution is $\tilde{f}_{Y}$ when the true distribution is $f_{Y}($ Cover \& Thomas, 1991).

The Kullback-Leibler divergence between two probability density distributions from $\tilde{f}_{Y}$ to $f_{Y}$ is defined as the expectation over the distribution $f_{Y}$, it is given by:

$$
D_{\mathrm{KL}}\left(f_{Y} \| \tilde{f}_{Y}\right)=E_{f_{Y}}\left[\log \frac{f_{Y}(y)}{\tilde{f}_{Y}(y)}\right]
$$

- $\quad$ For the discrete case:

$$
D_{\mathrm{KL}}\left(f_{Y} \| \tilde{f}_{Y}\right)=\sum_{y \in Y} f_{Y}(y) \log \frac{f_{Y}(y)}{\tilde{f}_{Y}(y)}
$$

- $\quad$ For the continuous case:

$$
D_{\mathrm{KL}}\left(f_{Y} \| \tilde{f}_{Y}\right)=\int f_{Y}(y) \log \frac{f_{Y}(y)}{\tilde{f}_{Y}(y)} d y
$$

\subsubsection{KLD for Multivariate Normal Distribution}

The multivariate normal distribution is a multivariate extension of the normal distribution of a single variable to a random vector of normally distributed elements, considering the correlation among those elements. 
Recalling that the Probability Density Function (PDF) for a normally distributed random variable $Y$, with mean $\mu$ and variance $\sigma^{2}$, is given by:

$$
f_{Y}(y)=\frac{1}{\sqrt{2 \pi} \sigma} \exp \left(-\frac{(y-\mu)^{2}}{2 \sigma^{2}}\right)
$$

In case of $n$-dimensional random vector $\boldsymbol{Y}=\left[\begin{array}{llll}\boldsymbol{y}_{1} & \boldsymbol{y}_{2} & \ldots & \boldsymbol{y}_{n}\end{array}\right]^{T}$, Equation Error! Reference source not found. is extended to take the form of:

$$
f_{Y}(\boldsymbol{y})=\frac{1}{(2 \pi)^{n / 2}}|\boldsymbol{\Sigma}|^{-1 / 2} \exp \left\{\frac{-\left(\boldsymbol{y}-\mu_{Y}\right)^{T} \boldsymbol{\Sigma}^{-1}\left(\boldsymbol{y}-\mu_{Y}\right)}{2}\right\}
$$

The notation $\boldsymbol{Y} \sim N_{n}(\boldsymbol{y} ; \mu, \boldsymbol{\Sigma})$ is used to denote the multivariate normal distribution of the $n$-dimension random vector $\boldsymbol{Y}$, where $\mu_{\boldsymbol{Y}}$ is the n-dimensional mean vector:

$$
\mu_{\boldsymbol{Y}}^{T}=E(\boldsymbol{Y})=\left[E\left(\boldsymbol{y}_{1}\right) E\left(\boldsymbol{y}_{2}\right) \ldots E\left(\boldsymbol{y}_{n}\right)\right]
$$

and $\boldsymbol{\Sigma} \in R^{n \times n}$ represents the symmetric positive definite covariance matrix, whose $(i, j)$ th entry is $\operatorname{Cov}\left[\boldsymbol{y}_{i}, \boldsymbol{y}_{j}\right]$. It is defined as:

$$
\boldsymbol{\Sigma}=\operatorname{Cov}(\boldsymbol{Y})=E\left[\left(\boldsymbol{Y}-\mu_{\boldsymbol{Y}}\right)\left(\boldsymbol{Y}-\mu_{\boldsymbol{Y}}\right)^{T}\right]=E\left[\boldsymbol{Y} \boldsymbol{Y}^{T}\right]-\mu_{\boldsymbol{Y}} \mu_{\boldsymbol{Y}}^{T}
$$

Having two multivariate normal distributions $f_{\boldsymbol{Y}}=N_{1}\left(\boldsymbol{y} ; \mu_{\boldsymbol{Y}_{1}}, \boldsymbol{\Sigma}_{1}\right)$ and $\tilde{f}_{\boldsymbol{Y}}=N_{2}\left(\boldsymbol{y} ; \mu_{\boldsymbol{Y}_{2}}, \boldsymbol{\Sigma}_{2}\right)$, the KL divergence between these distributions is:

$$
D_{\mathrm{KL}}\left(f_{\boldsymbol{Y}}|| \tilde{f}_{\boldsymbol{Y}}\right)=\frac{1}{2}\left\{\operatorname{tr}\left(\boldsymbol{\Sigma}_{2}^{-1} \boldsymbol{\Sigma}_{1}\right)+\left(\mu_{\boldsymbol{Y}_{2}}-\mu_{\boldsymbol{Y}_{1}}\right)^{T} \boldsymbol{\Sigma}_{2}^{-1}\left(\mu_{Y_{2}}-\mu_{\boldsymbol{Y}_{1}}\right)-n+\log \frac{\left|\boldsymbol{\Sigma}_{2}\right|}{\left|\boldsymbol{\Sigma}_{1}\right|}\right\}
$$

Where $\operatorname{tr}($.$) denotes the trace of a given matrix.$

\subsection{The Chi-square $Q-Q$ plot}

The Chi-square Q-Q plot (Korkmaz, et al., 2014) is used as a graphical tool to check the assumption of multivariate distribution for a given data. The squared Mahalanobis distance has an approximate chi-squared 
distribution for large samples of data that follows the multivariate normal distribution with $n$-degrees of freedom. The procedure includes the following steps:

1. Calculate the squared Mahalanobis distances to the centre of the points that have a chi-squared distribution with $p$-degrees of freedom as:

$$
M_{d}\left(\boldsymbol{y}^{k}\right)=\left(\boldsymbol{y}^{k}-\mu_{\boldsymbol{Y}}\right)^{T} \boldsymbol{\Sigma}^{-1}\left(\boldsymbol{y}^{k}-\mu_{\boldsymbol{Y}}\right)
$$

$\boldsymbol{y}^{k}=\left[y_{1, k}, y_{2, k}, \cdots, y_{n, k}\right]^{T}$ for $k=1: N_{s}$, where $N_{s}$ is the number of samples.

2. Arrange the distances in ascending order as $M_{d}^{1} \leq M_{d}^{2} \leq \cdots \leq M_{d}^{N_{S}}$

3. Compute the expected quantiles from the chi-squared distribution $X^{2}$ that correspond to the Mahalanobis distances as:

$$
q_{k}=X^{2}\left(\frac{k-1 / 2}{N_{s}}\right) \text { for } k=1: N_{s}
$$

4. The Chi-square Q-Q plot is then obtained by sketching the distances $M_{d}\left(\boldsymbol{y}^{k}\right)$ versus the quantiles $q_{k}$ for $k=1: N_{s}$

The plot resembles a straight line through the origin for data following multivariate normal distribution while a curved pattern indicates non-normality (Ramzan, et al., 2013).

\section{KLD based PCA Monitoring Scheme}

The proposed fault detection technique aims at detecting the presence of anomalies that cause process failures using KLD applied to a PCA model of the process data. The basic idea is based on computing the divergence between the multivariate probability density functions of the fault-free and faulty states of the scores through the calculation of KLD between the corresponding distributions. The block diagram in Figure 1 shows the main steps of our developed FD algorithm, the procedure was applied on both PCA subspaces, Principal Subspace 
(PS) and Residual Subspace (RS), resulting in two fault indicators entitled KLDPS and KLDRS. it can be summarized as:

1) PCA model subspaces $\boldsymbol{P}_{p c}$ and $\boldsymbol{P}_{r e s}$ (Equation 4) are identified from a training data set acquired under a healthy operating condition.

2) Reference score components matrices $\widehat{\boldsymbol{T}}_{\text {ref }}$ and $\widetilde{\boldsymbol{T}}_{\text {ref }}$ (Equations 6 and 7) are then obtained by projecting the fault-free data into both PCA subspaces. Subsequently, the reference probability distributions are established as $\tilde{f}_{\widehat{T}_{r e f}}$ and $\tilde{f}_{\widetilde{T}_{r e f}}$ (Equation 18):

$$
\begin{gathered}
\tilde{f}_{\widehat{T}_{r e f}}=\frac{1}{(2 \pi)^{l / 2}}\left|\Sigma_{\widehat{T}_{r e f}}\right|^{-1 / 2} \exp \left\{\frac{-\left(\boldsymbol{t}-\mu_{\widehat{T}_{r e f}}\right)^{T} \boldsymbol{\Sigma}_{\widehat{\boldsymbol{T}}_{r e f}}{ }^{-1}\left(\boldsymbol{t}-\mu_{\widehat{\boldsymbol{T}}_{r e f}}\right)}{2}\right\} \\
\tilde{f}_{\widetilde{\boldsymbol{T}}_{r e f}}=\frac{1}{(2 \pi)^{(m-l) / 2}}\left|\boldsymbol{\Sigma}_{\widetilde{\boldsymbol{T}}_{r e f}}\right|^{-1 / 2} \exp \left\{\frac{-\left(\boldsymbol{t}-\mu_{\widetilde{T}_{r e f}}\right)^{T} \Sigma_{\widetilde{T}_{r e f}}{ }^{-1}\left(\boldsymbol{t}-\mu_{\widetilde{T}_{r e f}}\right)}{2}\right\}
\end{gathered}
$$

where $\tilde{f}_{\widehat{\boldsymbol{T}}_{\text {ref }}}=N\left(\boldsymbol{t} ; \mu_{\widehat{\boldsymbol{T}}_{\text {ref }}}, \boldsymbol{\Sigma}_{\widehat{\boldsymbol{T}}_{\text {ref }}}\right)$ is estimated for the $l$ principal scores and $\tilde{f}_{\widetilde{\boldsymbol{T}}_{\text {ref }}}=N\left(\boldsymbol{t} ; \mu_{\widetilde{\boldsymbol{T}}_{r e f}}\right.$, $\left.\boldsymbol{\Sigma}_{\widetilde{\boldsymbol{T}}_{\text {ref }}}\right)$ for the $(m-l)$ residual scores evaluated for a window frame under healthy conditions.

3) The online scores $(\widehat{\boldsymbol{T}}$ and $\widetilde{\boldsymbol{T}})$ are calculated using the referenced PCA subspaces for the following set of measurements, and the corresponding probability density functions $f_{\widehat{T}}$ and $f_{\widetilde{T}}$ (Equation 18) are estimated at each time instant through a moving window.

4) KLD is calculated between the reference and the new probability densities according to Equation Error! Reference source not found. such that $\operatorname{KLDPS}=D_{\mathrm{KL}}\left(f_{\widehat{T}} \| \tilde{f}_{\widehat{T}_{r e f}}\right)$ and KLDRS $=$ $D_{\mathrm{KL}}\left(f_{\widetilde{T}} \| \tilde{f}_{\widetilde{T}_{r e f}}\right)$ are given by:

KLDPS $=\frac{1}{2}\left\{\operatorname{tr}\left(\boldsymbol{\Sigma}_{\widehat{\boldsymbol{T}}_{r e f}}^{-1} \boldsymbol{\Sigma}_{\widehat{T}}\right)+\left(\mu_{\widehat{\boldsymbol{T}}_{r e f}}-\mu_{\widehat{T}}\right)^{T} \boldsymbol{\Sigma}_{\widehat{\boldsymbol{T}}_{r e f}}^{-1}\left(\mu_{\widehat{\boldsymbol{T}}_{r e f}}-\mu_{\widehat{T}}\right)-l+\log \frac{\left|\boldsymbol{\Sigma}_{\widehat{\boldsymbol{T}}_{r e f}}\right|}{\left|\boldsymbol{\Sigma}_{\widehat{T}}\right|}\right\}$

$$
\operatorname{KLDRS}=\frac{1}{2}\left\{\operatorname{tr}\left(\boldsymbol{\Sigma}_{\widetilde{\boldsymbol{T}}_{r e f}}^{-1} \boldsymbol{\Sigma}_{\widetilde{T}}\right)+\left(\mu_{\widetilde{\boldsymbol{T}}_{r e f}}-\mu_{\widetilde{\boldsymbol{T}}}\right)^{T} \boldsymbol{\Sigma}_{\widetilde{\boldsymbol{T}}_{r e f}}^{-1}\left(\mu_{\widetilde{\boldsymbol{T}}_{r e f}}-\mu_{\widetilde{T}}\right)-(m-l)+\log \frac{\left|\boldsymbol{\Sigma}_{\widetilde{\boldsymbol{T}}_{r e f}}\right|}{\left|\Sigma_{\widetilde{T}}\right|}\right\}
$$


5) As a final point, the obtained KLDPS and KLDRS are compared to appropriate thresholds to infer the state of the process.

Notice that the healthy scores and their PDFs are calculated only once in the offline stage from sufficient training data, and their statistics (KLDPS, KLDRS) are used to determine the thresholds. On the contrary, the online scores are updated through the projection of any new measurement and collected in a moving window to estimate their current approximate PDFs and calculate their KLD values which are compared to the established thresholds.

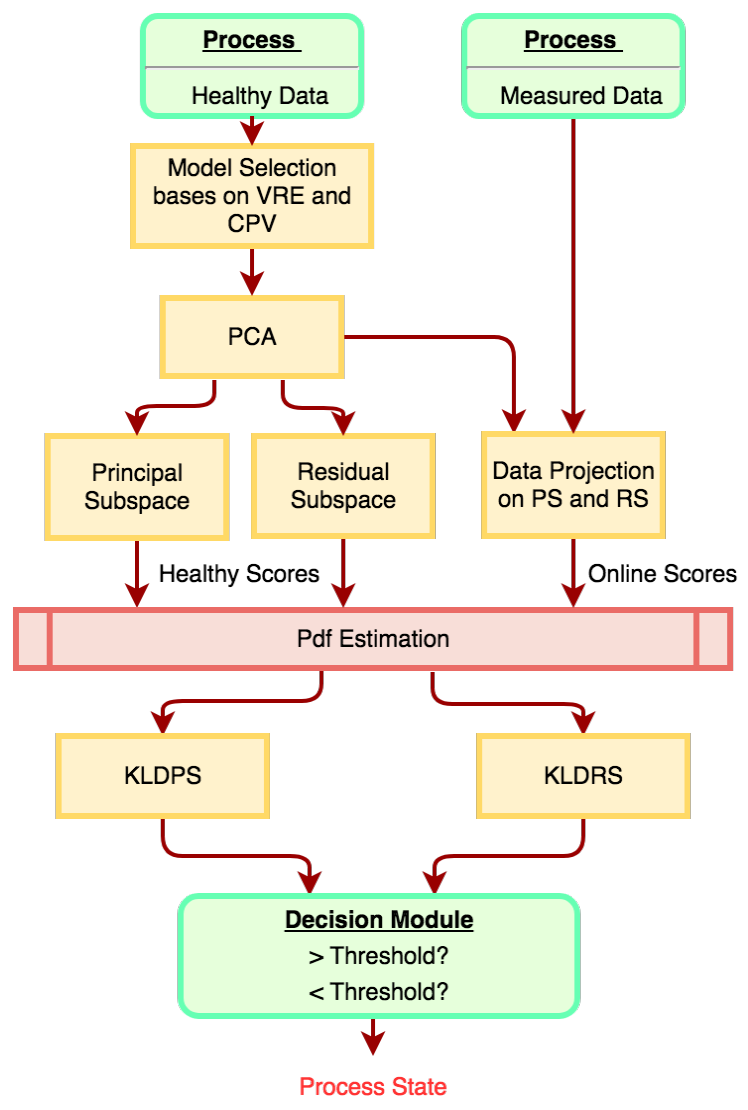

Figure 1 KLD-based PCA for fault detection procedure 


\section{Application}

Generally, MSPM techniques are tested and verified by application to certain industrial plants. Various data sets under specific faulty states can be collected from simulation or real operation in order to test the validity of the diverse proposed techniques. In this paper, two processes are used to testify the proposed method, the Tennessee Eastman Process (TEP) which is a simulated process and a real system composed of three tanks. TEP was chosen to be utilized in our work as it is an available realistic simulation program of a well-known plant in chemical engineering. It is widely accepted as a benchmark for evaluating process control and monitoring methods. Many research on process monitoring for fault detection and diagnosis have used the TEP as a source of data for comparing various approaches (Deng, et al., 2016; Rato \& Reis, 2013; Wang, et al., 2008). Similarly, the three tank system, consisting of tanks, pumps, and pipelines used in chemical industries, serves as typical benchmark process in control laboratories. The real data collected from the system and corrupted by faults is suitable to demonstrate the monitoring framework. The proposed FD method was verified by investigating the False Alarm Rate (FAR) and the Missed Detection Rate (MDR) defined as the probabilities of type I and type II errors, respectively (Ding, 2008; Zhang, et al., 2017a), in addition to the fault detection Time Delay (TD) for different data sets containing the fault-free operation part $(F=0)$ and faulty state part $(F \neq 0)$.

$$
\begin{gathered}
\mathrm{FAR} \%=\mathbb{P}\left(\mathrm{KLD}>\mathrm{KLD}_{\mathrm{th}} \mid F=0\right) \% \\
\mathrm{MDR} \%=\mathbb{P}\left(\mathrm{KLD}<\mathrm{KLD}_{\mathrm{th}} \mid F \neq 0\right) \% \\
\mathrm{TD}=t_{d}-t_{o}
\end{gathered}
$$

where $t_{d}$ is the fault detection time and $t_{o}$ is the fault occurrence time. 
The FAR reflects the robustness of the FD technique; the MDR quantifies the sensitivity to every fault that could possibly occur in the process, whereas the fault detection time delay of these faults defines the promptness of the FD system.

\section{Tennessee Eastman Process}

The TEP, proposed by Downs and Vogel as a benchmark for the Eastman Chemical Company, is well described in (Downs \& Vogel, 1993). the full flow diagram of the process is shown in Figure 2 (Chiang, et al., 2004). The measured variables include various measurements such as: pressures, levels, temperatures, concentrations, etc., while the manipulated variables include 11 valves' positions in addition to the reactor agitator speed.

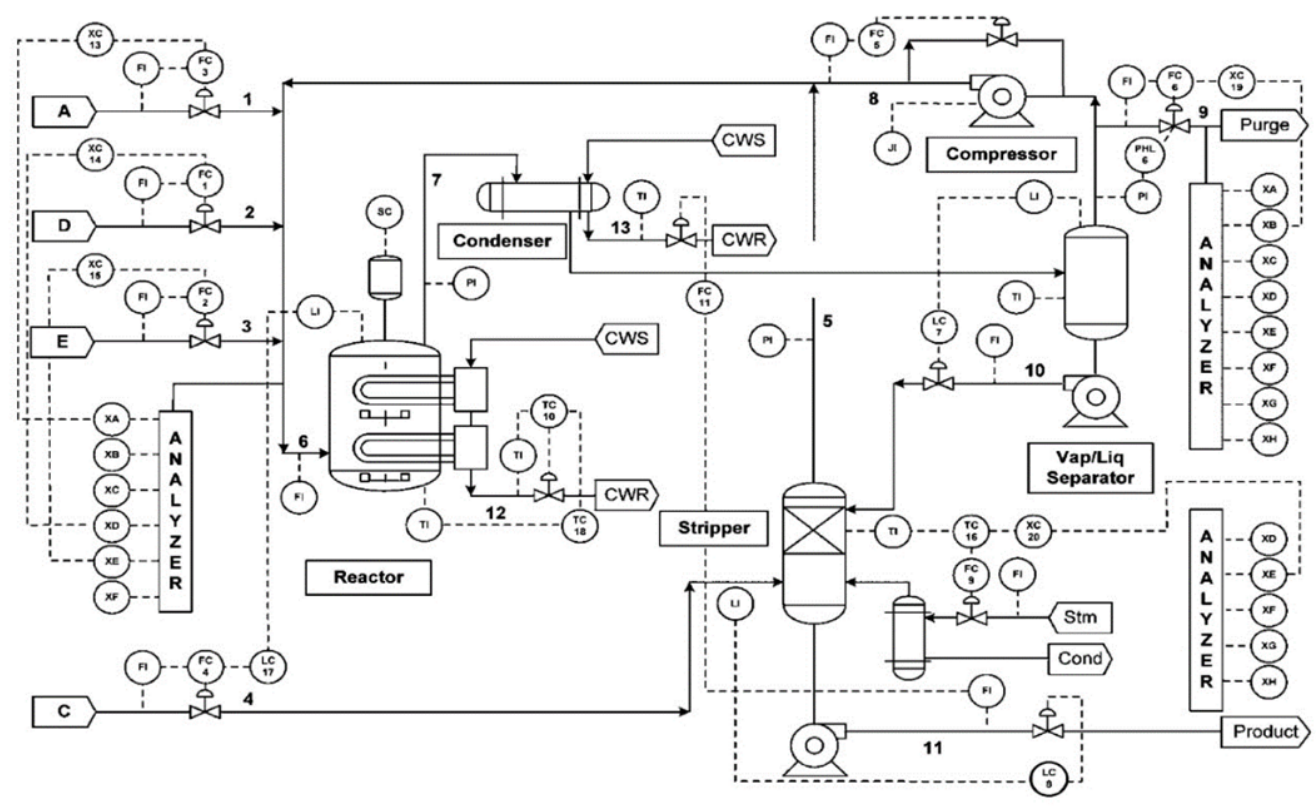

Figure 2 Tennessee Eastman diagram. (Chiang, et al., 2004)

A simulation code that imitates the real process was created by Downs and Vogel that was originally openloop unstable. For this application, a plant-wide control structure recommended using decentralized control open and closed multi-loop controllers. The MultiLoop_mode3.mdl model, designed to run at Mode 3 
conditions, is adopted to generate the simulated process data (Bathelt, et al., 2015). The simulation code was used to record 23 data sets of the process measurements for 130 operation hours. Two datasets correspond to a normal operating condition for training and testing purposes; the remaining 21 sets are faulty and each corresponds to a preprogrammed fault. These benchmark faults are listed in table 1 among which, 5 faults are of an unknown nature. In each faulty set, the fault is introduced after 40 operating hours. The sampling time was selected as $3 \mathrm{~min}$.

Table 1 Process faults for the Tennessee

\begin{tabular}{|c|c|c|}
\hline $\begin{array}{c}\text { Fault } \\
\text { Number }\end{array}$ & Description & Type \\
\hline 1 & A/C feed ratio, B composition constant (Stream4) & Step \\
2 & B composition, A/C ratio constant (Stream4) & Step \\
3 & D feed (Stream2) & Step \\
4 & Reactor cooling water inlet temperature & Step \\
5 & Condensor cooling water inlet temperature & Step \\
6 & A feed loss (Stream1) & Step \\
7 & Cheader pressure loss - reduced availability (Stream4) & Step \\
8 & A, B and C composition (Stream4) & Random \\
9 & D feed temperature (Stream2) & Random \\
10 & C feed temperature (Stream4) & Random \\
11 & Reactor cooling water inlet temperature & Random \\
12 & Condensor cooling water inlet temperature & Random \\
13 & Reaction kinetics & Slow drift \\
14 & Reactor cooling water valve & Sticking \\
15 & Condensor cooling water valve & Sticking \\
$16-20$ & Unknown & - \\
21 & Stream 4 valve & Constant position \\
\hline
\end{tabular}

\subsection{Analysis}

The KLD fault indicator is evaluated for the principal and the residual subspaces obtained from the PCA model of the TEP. Each subspace scores can be thought of as random vectors of independent identically distributed (iid) random variables. The illustration of the divergence concept will be shown later with the three tank system where the number of variables is small since it is impossible to visualize a changing $n$-variate distribution using demonstration tools for $n>2$. Nevertheless, the assessment of a heavy assumption considering the multivariate normality of the scores is first provided in the following. Figure 3 shows the Chisquare Q-Q plot as described in subsection 2.4. This normality test is verified for the training samples of the 
principal as well as the residual score subspaces ( $\widehat{\boldsymbol{T}}$ (Equation 6) and $\widetilde{\boldsymbol{T}}$ (Equation 7)) of dimensions $N_{t r} \times l$ and $N_{t r} \times(m-l)$, respectively, where $N_{t r}$ is the number of measurements in the training dataset. Figure 3 indicates that the majority of the points in the graph, defined by the Mahalanobis distance (Equation 22) versus the Chi-square quantile (Equation 23), tend to fall into the reference line which proves that the multivariate normality assumption holds. Only few points exhibit a negligible deviation which is acceptable and it is attributed to measurement noise.

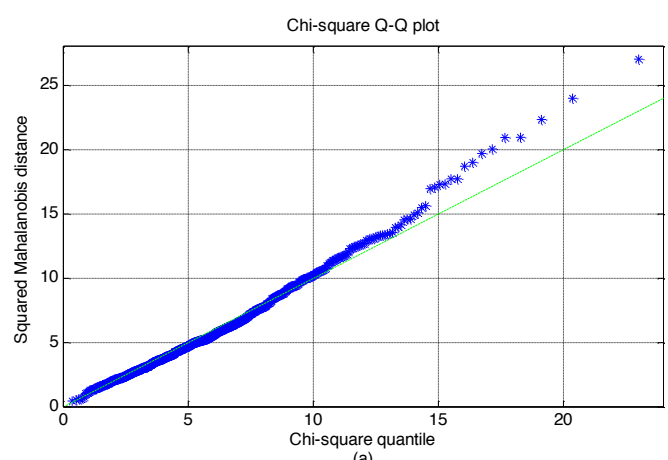

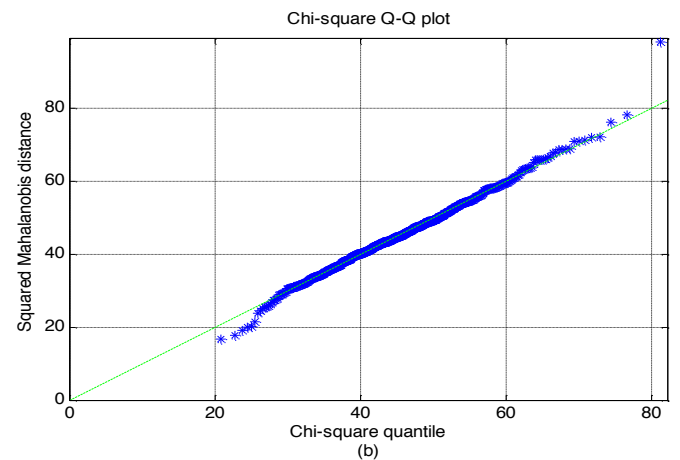

Figure 3 Chi-square Q-Q plot for assessing multivariate normality of (a) the principal scores and (b) the residual scores

\subsection{TEP fault monitoring}

In this section, the proposed algorithm of KLD-based PCA is applied for FD in Tennessee Eastman process. The algorithm is tested and evaluated by investigating False Alarm Rate (FAR), Missed Detection Rate (MDR) (Zhang, et al., 2017a) and the fault detection Time Delay (TD) for data being collected for the 21 benchmark faults. The developed method is also tested on different PCA models based on two different criteria. The number of retained components in the PCA model (Equations 3 and 4) was found to be 6 scores when using the VRE method (Equations (9 to 12)), while 37 scores are retained if a 90\% CPV is used instead (Equation (13)). Despite the huge difference in the appropriate number of PCs (6 vs 37), which stands for an unsolved dilemma where each of the different criteria is claimed to be the optimum (Zwick \& Velicer, 1989), the developed algorithm is less dependent on the exact choice of $l$ according to its overall performance. The window size, another parameter that should be tuned, was set by testing the data for minimum average MDR and FAR in order to 
have a large enough window length that ensures the obtained densities at a given time are really descriptive to the local distributions. For the PCA model based on VRE, the window size was 630 samples for the KLDPS and 660 samples for KLDRS. The same approach was followed for the subspaces obtained from the CPV method giving that the best window size for the KLDPS is 690 samples versus 510 samples for the KLDRS. As expected, the window length is relatively related to the subspace dimension and the amount of captured noise. The two monitoring statistics are evaluated over a moving window that includes current and most recent samples. The use of a moving window approach gives more importance to the local information while disregarding old observations, this generally makes the monitoring more effective (Russell, et al., 2000). Moreover, with no update on PCA model parameters, this approach avoids an unnecessary increase in the computational complexity with a fixed PCA model. More importantly, the moving window approach allows the estimation of local density functions (Equations 18, 19, and 20) of both subspaces of the scores at any given time. The offline training data is then used as in an online approach and scanned with a moving window, the KLD is evaluated for each window with respect to the reference distribution; The resulting collections of the KLDRS and KLDPS, as described in section 3, are used to define their proposer thresholds during the given healthy operation conditions. The thresholds were established statistically based on the chosen confidence level. It consisted of alignment of the detection thresholds to correspond to a $1 \%$ FAR that stands for the effects of noise and mismatches while increasing the sensitivity. This tuning is achieved through the training data that corresponds to normal operating conditions by using the percentile approach for limits correction (Aldrich \& Auret, 2013). Zero FAR was achieved using both statistics on the testing data which proves the high robustness of the FD system.

The performance parameters related to the 21 faulty sets are calculated for both fault indicators and summarized in Table 2. (where und refers to undetected faults)

Recall that the most robust FD scheme is the one that minimizes the FAR as defined by Equation (28), while the most effective FD scheme is the one associated with the lowest MDR defined by Equation (29) and the 
shortest TD by Equation (30) which are sensitivity parameters. The most reliable FD scheme is therefore selected to be the one associated with the lowest parameters reported in Table 2. Corresponding to $99 \%$ confidence level, Table 2. shows the performance of KLDPS and KLDRS statistics in detecting different faults,

\begin{tabular}{|c|c|c|c|c|}
\hline & \multicolumn{4}{|c|}{ FAR (\%)/MDR (\%)/TD(samples) } \\
\hline & \multicolumn{2}{|c|}{ VRE 6 PCs } & \multicolumn{2}{|c|}{$90 \%$ CPV 37 PCs } \\
\hline & KLDPS Statistic & KLDRS statistic & KLDPS Statistic & KLDRS statistic \\
\hline IDV1 & $0 / 1.17 / 21$ & $0 / 0.67 / 12$ & $0 / 1.11 / 19$ & $0 / 0.39 / 7$ \\
\hline IDV2 & $0 / 3.22 / 58$ & $0 / 3.89 / 70$ & $0 / 4.77 / 86$ & $0 / 2.83 / 51$ \\
\hline IDV3 & $0 / 84.79 / 422$ & $0 / 19.99 / 350$ & $0 / 34.37 / 463$ & $0 / 6.55 / 118$ \\
\hline IDV4 & $0 / 9.55 / 172$ & $0 / 0.22 / 4$ & $0 / 2.22 / 40$ & $0 / 0.17 / 3$ \\
\hline IDV5 & $4.09 / 0 / 0$ & $0 / 0.06 / 1$ & $0 / 0.06 / 1$ & $0 / 0.06 / 1$ \\
\hline IDV6 & $0 / 7.2 / 9$ & $0 / 0.8 / 1$ & $0 / 9.6 / 12$ & $0 / 0.8 / 1$ \\
\hline IDV7 & $0 / 0.17 / 3$ & $0 / 0.06 / 1$ & $0 / 0.28 / 5$ & $0 / 0.06 / 1$ \\
\hline IDV8 & $0 / 1.61 / 29$ & $0 / 0.83 / 15$ & $0 / 1.44 / 26$ & $6.87 / 0.67 / 12$ \\
\hline IDV9 & $0 / 81.45 / 333$ & $0 / 29.48 / 429$ & $0 / 81.23 / 454$ & $0 / 14.05 / 253$ \\
\hline IDV10 & $0 / 7.50 / 135$ & $0 / 2.22 / 40$ & $0 / 9.05 / 163$ & $0 / 1.72 / 31$ \\
\hline IDV11 & $0 / 8.88 / 160$ & $0 / 0.67 / 12$ & $0 / 1.22 / 22$ & $0 / 0.61 / 11$ \\
\hline IDV12 & $0 / 0.22 / 4$ & $0 / 0.17 / 3$ & $0 / 0.22 / 4$ & $0 / 0.11 / 2$ \\
\hline IDV13 & $0 / 9.55 / 172$ & $0 / 6.55 / 118$ & $0 / 8.60 / 155$ & $0 / 6.61 / 119$ \\
\hline IDV14 & $0 / 16.10 / 290$ & $0 / 0.50 / 9$ & $0 / 1.05 / 19$ & $0 / 0.44 / 8$ \\
\hline IDV15 & 0/100/und & 0/100/und & 0/100/und & $0 / 72.4 / 358$ \\
\hline IDV16 & 0/100/und & $0 / 96.72 / 621$ & 0/100/und & $5.79 / 29.09 / 186$ \\
\hline IDV17 & $0 / 1.99 / 36$ & $0 / 1.11 / 20$ & $0 / 1.50 / 27$ & $0 / 0.83 / 15$ \\
\hline IDV18 & $0 / 3.72 / 67$ & $0 / 3.16 / 57$ & $0 / 3.55 / 64$ & $0 / 2.89 / 52$ \\
\hline IDV19 & $0 / 1.33 / 24$ & $0 / 0.94 / 17$ & $0 / 1.28 / 23$ & $0 / 0.72 / 13$ \\
\hline IDV20 & $0 / 6.27 / 113$ & $0 / 5.44 / 98$ & $0 / 6.55 / 118$ & $0 / 5.1 / 92$ \\
\hline IDV21 & 0/100/und & 0/100/und & 0/100/und & 0/100/und \\
\hline Avr MDR & 25.94 & 17.78 & 22.29 & 11.72 \\
\hline
\end{tabular}

in terms of the FAR, MDR and fault detection TD. According to Russel, et al. (2000) and Zhang (2009), detection of faults $3,9,15,16$ and 21 is very difficult because there are no noticeable changes in the observations. This problem has been overcome to an appreciable extent for faults 3 and 9 by considering the use of both statistics. However, fault 15 was detected by the KLDRS of the retained components from the CPV analysis. Furthermore, the detection of fault 16 was improved upon by both KLDRS statistic. These improvements may be considered as proof of the efficacy of the proposed method.

Taking an overview of the obtained results leads to a preliminary conclusion that the proposed FD system can be considered as a suitable technique for detection. The KL divergence statistics are sensitive to the 
abnormality since they introduce considerably low values for the factors characterizing the FD system performance which is the case for most of the faults with high robustness to false alarms. Also, it is noted that the CPV method for separating the two subspaces gave better results of the performance parameters than the VRE technique due to the suitable separation between the subspaces. 

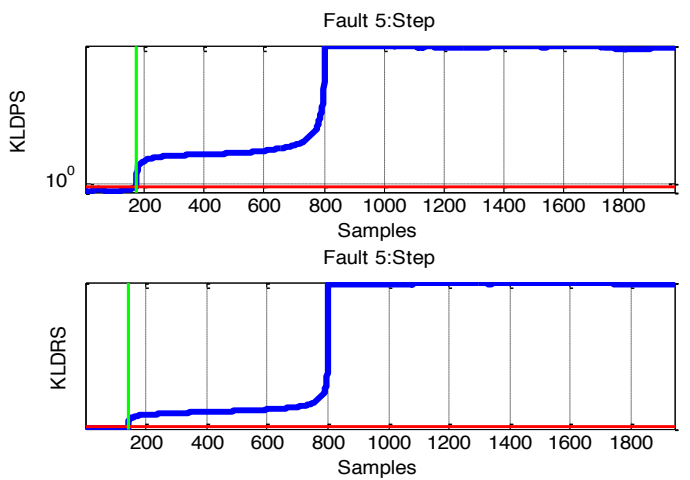

Figure 4 Monitoring results for fault 5 using KLDPS and KLDRS at 99\% CL obtained from the VRE technique
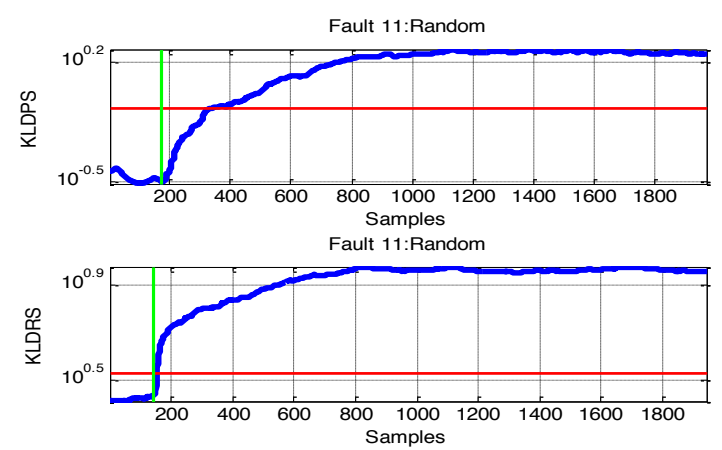

Figure 6 Monitoring results for fault 11 using KLDPS and KLDRS at 99\% CL obtained from the VRE technique
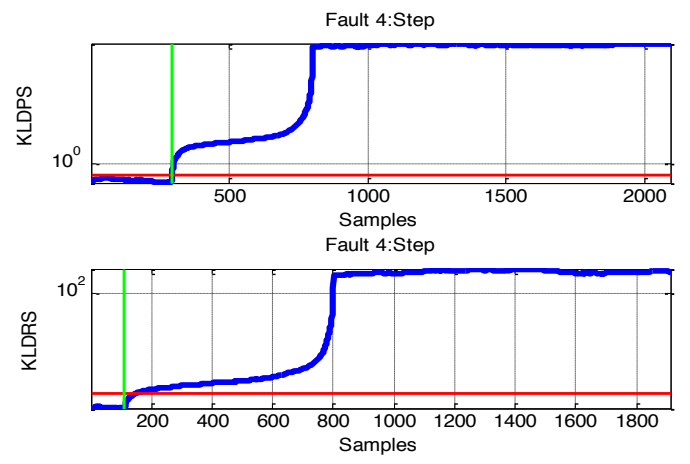

Figure 8 Monitoring results for fault 4 using KLDPS and KLDRS at 99\% CL obtained from the CPV technique
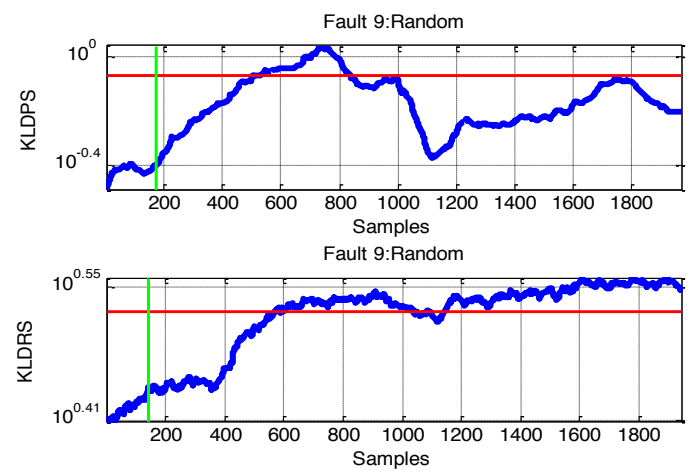

Figure 5 Monitoring results for fault 9 using KLDPS and KLDRS at 99\% CL obtained from the VRE technique

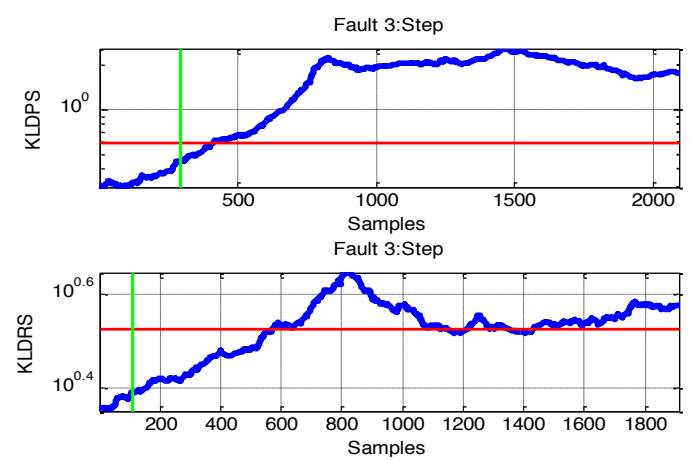

Figure 7 Monitoring results for fault 3 using KLDPS and KLDRS at 99\% CL obtained from the CPV technique
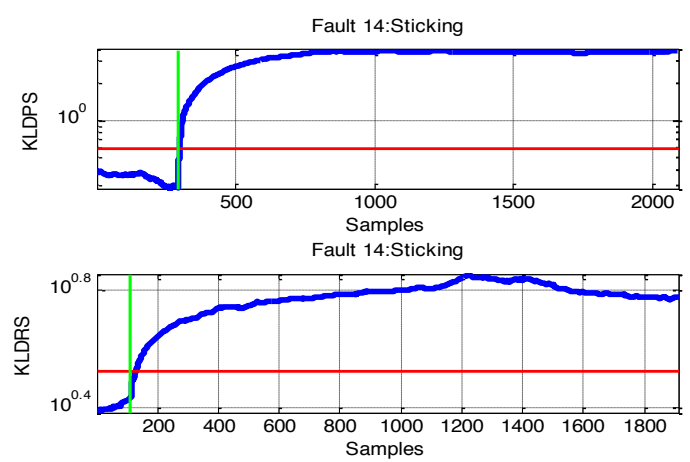

Figure 9 Monitoring results for fault 14 using KLDPS and KLDRS at $99 \% \mathrm{CL}$ obtained from the CPV technique 
To visualize these results and confirm the supposition of efficiency, different figures were plotted that show process faulty state operation monitoring using the two fault indicators for 6 different faults. The horizontal red line corresponds to $99 \%$ confidence level threshold and the vertical green line indicates the fault occurrence time.

For the statistics obtained from the VRE technique process faulty operation monitoring for the step fault 5 and the random faults 9 and 11 are shown in Figure 4, 5 and 6, respectively, while the step faults 3 and 4 are depicted in Figure 7 and Figure 8 and the sticking fault 14 in Figure 9 for the analysis done using CPV method. Process faults can affect one indicator differently with respect to the other. In both cases, employing VRE and CPV, the KLDRS gave better results than the KLDPS for almost all the faults. This can be observed from Figure 6 and Figure 9 where both faults 11 and 14 are promptly detected by KLDRS better than the KLDPS. This is also clearly noticed in Figure 5 and Figure 7 in which Faults 3 and 9 are barely detected using KLDPS while KLDRS was capable of providing satisfactory results. As a result, the KLDRS in the proposed methodology is considered to be the best fault indicator since it provides optimal performance factors for an adequate FD system.

\subsection{Comparison study on TEP}

A comparative study is conducted between multivariate data analysis methods results from Yu (2016) and Zhang, et al. (2017b) and those obtained from our proposed method with 99\% confidence level to show its proficiency and advantages. This FD statistics comparison is pursued in terms of the missed detection rate as listed in Table 3 where the minimum MDR is highlighted in red boldface.

The MDR values of each method are nearly similar in the cases of faults $1,2,6,7,8,12,13$ and 14 since the values are so small that they can be easily detected. A high percentage of the MDR indicates the undetectability of a certain fault which is noticeable in faults 15 for all the listed FD methods. The noteworthy improvement that the proposed method makes can be seen in reducing significantly the values of the MDR percentage of other faults specifically for faults 3 and 9 which present, in general, a weak spot for most statistical 
FD techniques, proving the KLD capacity of detecting small process shifts that are difficult to detect. Process faults that must be pointed out to are faults 15,17 and 18 where the lowest values of the MDR are achieved using the KLDRS. These results indicate the high sensitivity of the proposed technique to the process faults. In general, KL divergence shows a better performance compared to the MMP-based D statistic, LGPCA-based D and SPE statistics, LGP, $\mathrm{T}^{2}$ using PLS and DDPLS and DPLC-ICA approach. Additionally, the total average of MDR was computed for all the methods and the KL divergence-based monitoring provides the best MDR average value among all the FD techniques by considering the KLDRS fault indicator.

Table 3 MDR (\%) of MMP, JLGLPP, LGP, PLS, DDPLS, DPLC-ICA and the proposed method (KLDPS and KLDRS)

\begin{tabular}{|c|c|c|c|c|c|c|c|c|c|}
\hline & \multicolumn{4}{|c|}{ Jianbo Yu } & \multicolumn{3}{|c|}{ Kai Zhang et.al. } & \multirow{2}{*}{\multicolumn{2}{|c|}{$\begin{array}{c}\text { Proposed method } \\
\text { KLD-PCA }\end{array}$}} \\
\hline & \multirow{2}{*}{$\begin{array}{c}\text { MMP } \\
\text { D } \\
\end{array}$} & \multicolumn{2}{|c|}{ JLGLPP } & \multirow[t]{2}{*}{ LGP } & \multirow{2}{*}{$\begin{array}{c}\text { PLS } \\
T^{2}\end{array}$} & \multirow{2}{*}{$\begin{array}{c}\text { DDPLS } \\
\mathbf{T}^{2} \\
\end{array}$} & \multirow[t]{2}{*}{$\begin{array}{c}\text { DPLC- } \\
\text { ICA }\end{array}$} & & \\
\hline & & D & SPE & & & & & KLDPS & KLDRS \\
\hline IDV1 & 0 & 0 & 1 & 0 & 0.4 & 0.2 & 0 & 1.11 & 0.39 \\
\hline IDV2 & 3 & 2 & 2 & 2 & 1.8 & 1.2 & 0 & 4.77 & 2.83 \\
\hline IDV3 & 99 & 93 & 99 & 89 & 100 & 100 & 100 & 34.37 & 6.55 \\
\hline IDV4 & 28 & 76 & 40 & 55 & 100 & 100 & 100 & 2.22 & 0.17 \\
\hline IDV5 & 0 & 0 & 76 & 0 & 100 & 100 & 100 & 0.06 & 0.06 \\
\hline IDV6 & 0 & 0 & 0 & 0 & 0.6 & 0 & 0 & 9.6 & 0.8 \\
\hline IDV7 & 0 & 3 & 0 & 0 & 0 & 0 & 0 & 0.28 & 0.06 \\
\hline IDV8 & 13 & 2 & 3 & 2 & 5.7 & 1.3 & 0 & 1.44 & 0.67 \\
\hline IDV9 & 99 & 96 & 99 & 95 & 100 & 100 & 100 & 81.23 & 14.05 \\
\hline IDV10 & 17 & 9 & 63 & 6 & 23.8 & 12.8 & 1.2 & 9.05 & 1.72 \\
\hline IDV11 & 53 & 69 & 45 & 33 & 20.4 & 5 & 0.6 & 1.22 & 0.61 \\
\hline IDV12 & 2 & 0 & 2 & 0 & 49 & 34 & 12.4 & 0.22 & 0.11 \\
\hline IDV13 & 5 & 5 & 6 & 5 & 100 & 100 & 100 & 8.6 & 6.61 \\
\hline IDV14 & 0 & 2 & 0 & 0 & 11.6 & 0 & 0 & 1.05 & 0.44 \\
\hline IDV15 & 99 & 89 & 97 & 88 & 100 & 100 & 100 & 100 & 72.4 \\
\hline IDV16 & 12 & 12 & 80 & 9 & 100 & 100 & 100 & 100 & 29.09 \\
\hline IDV17 & 6 & 6 & 18 & 4 & 14.4 & 11.2 & 3.7 & 1.5 & 0.83 \\
\hline IDV18 & 10 & 10 & 11 & 10 & 23.2 & 13.6 & 13.4 & 3.55 & 2.89 \\
\hline IDV19 & 13 & 11 & 98 & 10 & 1.7 & 1.7 & 0.5 & 1.28 & 0.72 \\
\hline IDV20 & 16 & 9 & 59 & 9 & 7.8 & 8.4 & 1.9 & 6.55 & 5.1 \\
\hline IDV21 & 88 & 40 & 62 & 42 & 100 & 100 & 100 & 100 & 100 \\
\hline Av MDR & 26.81 & 25.42 & 41 & 21.85 & 45.73 & 42.35 & 39.7 & 22.29 & 11.72 \\
\hline
\end{tabular}




\section{Three tank system}

The three tank system introduced in this paper is the laboratory setup DTS200 (AMIRA-DTS200, 1996). The system consists of three tanks interconnected by two pipes that are equipped by two transfer valves (transfer valve 1 and 3). Two pumps are used to supply the water from the central reservoir to tank 1 and 2 with flow rates $q_{1}$ and $q_{2}$, respectively. The water levels at the three tanks are measured through piezo-resistive pressure transducers (level sensor $L_{1}, L_{2}$ and $L_{3}$ ). The observations provided from the flow rates and water levels are acquired using NI DAQ-6024E acquisition card interfaced with MATLAB software. The collected healthy data set contains 9000 samples while the faulty sets contain 2400 samples with sampling time equals to 0.2 seconds, each of 5 variables corresponding to the three tanks water levels $\left(L_{1}, L_{2}, L_{3}\right)$ and the two pump flows $\left(q_{1}, q_{2}\right)$ (Kouadri, et al., 2013).

\subsection{Analysis and results}

The aforementioned criterion, CPV, was applied to determine the number of retained PCs since it provided better results than VRE in the TEP. The results revealed 3 principal components expressing $90 \%$ of CPV to define the split between the principal and residual subspaces. Hence, the residual subspace contains two variables; to enhance the concept of distribution disparity, an inclusive example is used to illustrate the principle of our proposed technique. Figure 10 displays the difference between the distributions before and after fault occurrence using the two score components obtained from the residual subspace of the three tank system.

The window size, on the other hand, was set by testing the data for minimum average MDR and FAR. The window size was found to be 350 samples for KLDPS and 450 samples for KLDRS. Lastly, the thresholds were established statistically based on $99 \%$ confidence level according to the same principle adopted earlier.

The data gathered from the laboratory three tank system introduce two cases of sensor faults:

Case 1: 10\% of failure on the sensor level 1 occurring at sample 653,

Case 2: $10 \%$ of failure on the sensor level 3 occurring at sample 901. 


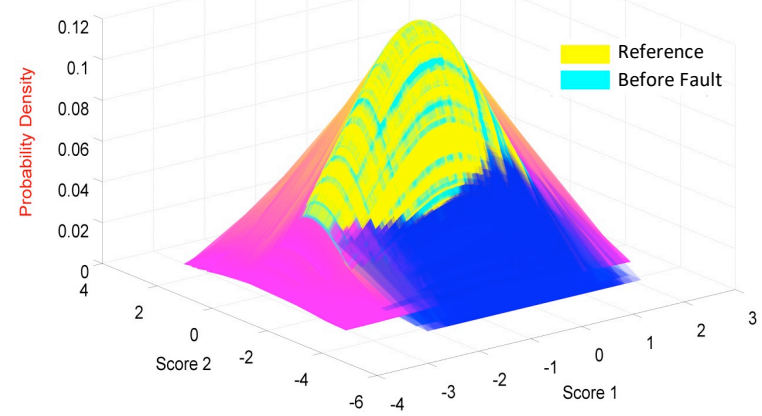

(a)

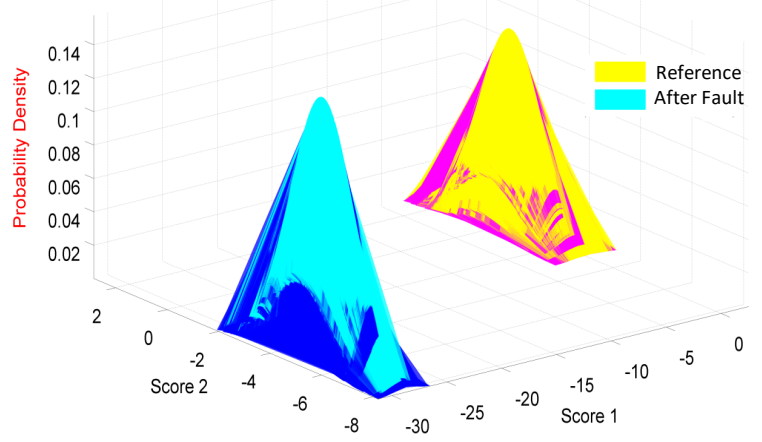

(b)

Figure 10 Comparison between PDFs of bivariate scores before (a) and after (b) fault occurrence obtained from the residual subspace of the three tank system in fault case 1

The performance indices (MDR and FAR) corresponding to the two fault modes are computed for both fault indicators (KLDPS and KLDRS) and summarized in table 4 along with the results obtained through the static PCA control charts ( $\mathrm{T}^{2}$ and SPE). It is noticeable that the proposed framework can be considered as a suitable technique for fault detection. The sensitivity of the KLD statistics to abnormal events is readily discernible through the values of MDR and FAR recorded. Furthermore, it is noted from the stated table that KLDRS gave better results of the performance parameters than the other statistics especially for fault case 2 , which confirms the superiority of KLDRS concluded before from the TEP. In order to visualize the results, the monitoring plots were depicted using the proposed method and static PCA for the two faults cases (Figure 11, 12, 13 and 14). Comparing the probability distributions of the online observations with the training ones through KullbackLeibler divergence proved its efficiency by achieving the trade-off between the detectability of failures and the false alarm rates. 
Table 4 MDR (\%) and FAR (\%) of static PCA and the proposed method (KLDPS and KLDRS)

\begin{tabular}{|c|c|c|c|c|c|c|c|c|}
\cline { 2 - 9 } \multicolumn{1}{c|}{} & \multicolumn{4}{c|}{ KLD-PCA } & \multicolumn{3}{c|}{ Static PCA } \\
\cline { 2 - 10 } \multicolumn{1}{c|}{ KLDPS } & \multicolumn{2}{c|}{ KLDRS } & \multicolumn{3}{c|}{ T $^{\mathbf{2}}$} & \multicolumn{2}{c|}{ SPE } \\
\hline Case & FAR(\%) & MDR(\%) & FAR(\%) & MDR(\%) & FAR(\%) & MDR(\%) & FAR(\%) & MDR(\%) \\
\hline $\mathbf{1}$ & 17.43 & 0 & $\mathbf{0 . 9 8}$ & $\mathbf{0}$ & 22.24 & 0 & 9.35 & 0 \\
\hline $\mathbf{2}$ & 0 & 100 & $\mathbf{0}$ & $\mathbf{1 1 . 9 5}$ & 4.45 & 93.67 & 8.01 & 79.81 \\
\hline
\end{tabular}
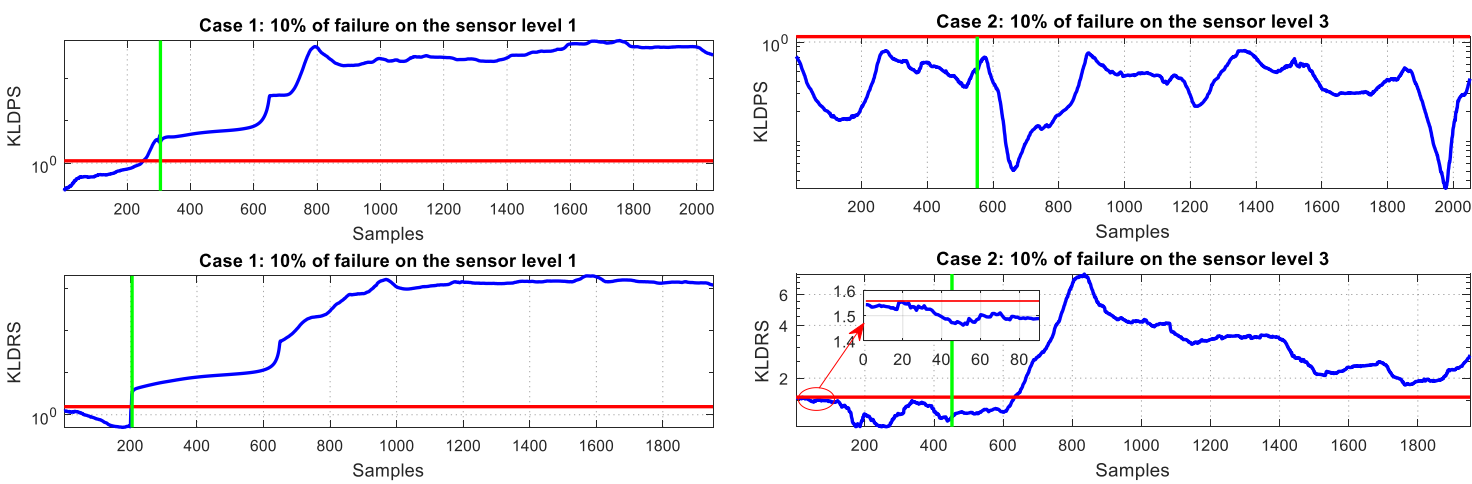

Figure 11 Monitoring results of fault case 2 using KLDPS and KLDRS at $99 \%$ CL

Figure 12 Monitoring results of fault case 2 using KLDPS and KLDRS at $99 \%$ CL
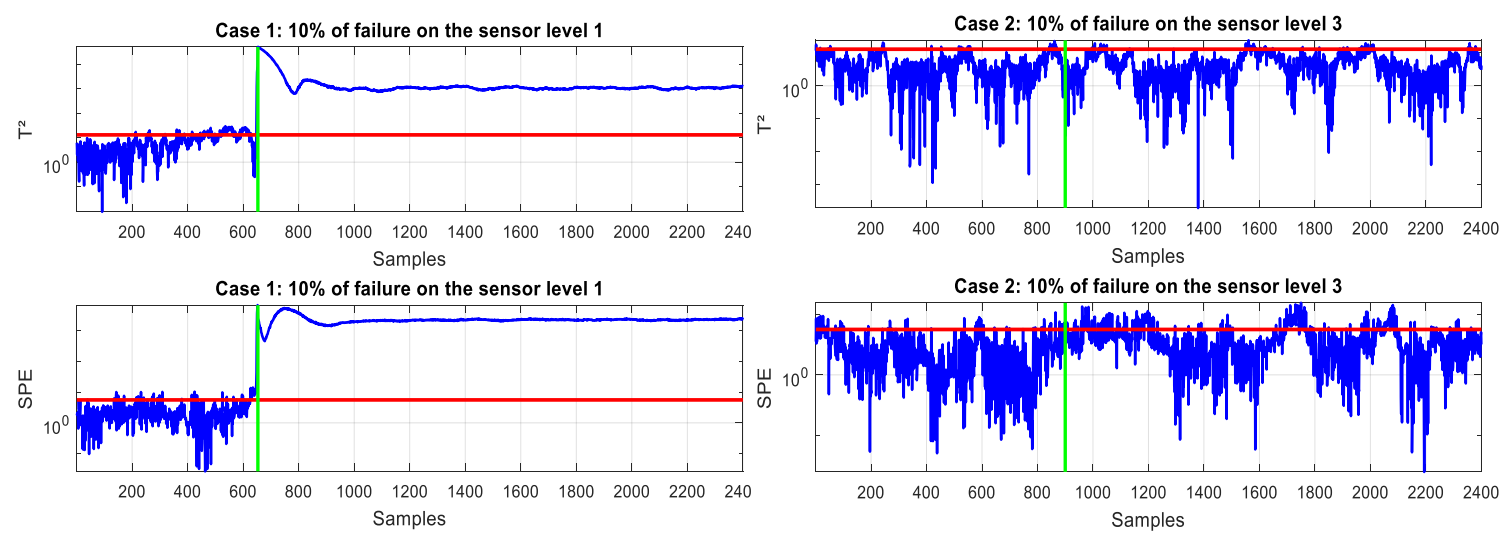

Figure 13 Monitoring results of fault case 1 using $\mathrm{T}^{2}$ and SPE at $99 \% \mathrm{CL}$

Figure 14 Monitoring results of fault case 2 using $\mathrm{T}^{2}$ and SPE at $99 \% \mathrm{CL}$

\section{Conclusion}

A probabilistic measure, called the Kullback-Leibler Divergence (KLD) based on PCA is introduced in this paper. PCA was used as a modeling framework describing data variation in the principal and the residual 
subspaces. The obtained scores of both subspaces follow a multivariate Gaussian distribution and their approximate PDFs were sequentially estimated in a moving window approach then evaluated through the KLD which measures all kinds of dissimilarity between the probability distributions among the cores in both subspaces during normal and faulty operating modes. Instead of the traditional squared distance charts such as $Q$ and $T^{2}$ statistics, more accurate fault indicators are developed for a robust and sensitive measure of deviation within the two PCA-defined subspaces.

The developed methods were first validated through the well-known TEP benchmark and then put to a realistic challenge in an experimental application against real faults using real data. The obtained results revealed that the proposed method has aptly proven its capability and efficacy in detecting several faults in a simulated as well as realistic processes as it afforded minimum values of the performance factors, namely the MDR, the FAR and the FD time delay that are mostly used as standard measurements characterizing the utility and the validity of the FD approach.

Another approach that was followed within the study is the comparison between the KLD-based PCA and some other MSPM methods for FD including MMP, JLGLPP, LGP, PLS, DDPLS, and DPLC-ICA. The idea was handled by comparing the Hotelling's $\mathrm{T}^{2}$, SPE and D statistics to the developed KLDPS and KLDRS statistics.

As future work, we suggest extending the proposition of using the KLD based PCA algorithm to other industrial processes subject to different types of faults. Also, the disparity measure can be obtained by the use of other MSPM methods such as the PLS. Moreover, the technique in our work discussed only the fault detection phase, hence, the KLD is recommended to be set into a classification analysis in which the complete process of fault detection and isolation is to be considered.

\section{References}


Aggoune, L., Chetouani, Y. \& Raïssi, T., 2016. Fault detection in the distillation column process using Kullback Leibler divergence. ISA Trans., Volume 63, pp. 394-400.

Alcorta García, E. \& Frank, P., 1997. Deterministic nonlinear observer-based approaches to fault diagnosis: A survey. Control Eng. Prac., 5(5), pp. 663-670.

Aldrich, C. \& Auret, L., 2013. Unsupervised Process Monitoring and Fault Diagnosis with Machine Learning Methods. London: Springer.

AMIRA-DTS200, 1996. Laboratory Experiment Three Tank System. Duisburg: Amira GmbH.

Bathelt, A., Ricker, N. L. \& Jelali, M., 2015. Revision of the Tennessee Eastman process model. IFACPapersOnLine, 48(8), pp. 309-314.

Chen, J. \& Patton, R. J., 1999. Robust Model-Based Fault Diagnosis for Dynamic Systems. Boston, MA: Springer US.

Chiang, L. H., Kotanchek, M. E. \& Kordon, A. K., 2004. Fault diagnosis based on Fisher discriminant analysis and support vector machines. Comput. Chem. Eng., 28(8), pp. 1389-1401.

Cover, T. \& Thomas, J., 1991. Entropy, Relative Entropy and Mutual Information. In: Elements of Information Theory. s.1.: John Wiley \& Sons, Inc.

Deng, X., Tian, X., Chen, S. \& Harris, C., 2016. Nonlinear process fault diagnosis based on serial principal components analysis. IEEE Trans. Neural Netw. Learn. Syst., pp. 1-13.

Ding, S., 2008. Model-based Fault Diagnosis Techniques: Design Schemes, Algorithms, and Tools. s.1.: Springer.

Downs, J. J. \& Vogel, E. F., 1993. A plant-wide industrial process control problem. Comput. Chem. Eng., Volume 17, pp. 245-255.

Dunia, R., Qin, . S. J., Edgar, T. F. \& McAvoy, T. J., 1996. Identification of faulty sensors using principal component analysis. AIChE J., Volume 42, pp. 2797-2812. 
Dunia, R. \& Qin, S., 1998. Joint diagnosis of process and sensor faults using principal component analysis. Control Eng. Pract., Volume 6, pp. 457-469.

Frank, P. M., Ding, S. X. \& Marcu, T., 2000. Model-based fault diagnosis in technical processes. T.I. Meas. Cont., 22(1), pp. 57-101.

Gertlert, J., Li, W., Huang, Y. \& McAvor, T., 1999. Isolation enhanced principal component analysis. AIChE $J ., 45(2)$, pp. 323-334.

Guo, R., Zhang, N., Wang, J. \& Dong, J., 2018. Phase partition and identification based on a two-step method for batch process. T.I. Meas. Control, 40(16), pp. 4472-4483.

Harkat, M., Mourot, G. \& Ragot, J., 2000. Sensor failure detection of air quality monitoring network. IFAC Proc. Vol., Volume 33, pp. 529-534.

Harmouche, J., Delpha, C. \& Diallo, D., 2014. Incipient fault detection and diagnosis based on KullbackLeibler divergence using principal component analysis: Part I. Signal Process., Volume 94, pp. 278-287.

Harmouche, J., Delpha, C. \& Diallo, D., 2015. Incipient fault detection and diagnosis based on KullbackLeibler divergence using principal component analysis: Part II. Signal Process., Volume 109, pp. 334-344.

Hauck, D. J., Runger, G. C. \& Montgomery, D. C., 1999. Multivariate statistical process monitoring and diagnosis with grouped regression-adjusted variables. Commun. Stat. Simul. Comput., 28(2), pp. 309-328.

Huang, J. \& Yan, X., 2015. Dynamic process fault detection and diagnosis based on dynamic principal component analysis, dynamic independent component analysis and Bayesian inference. Chemometr. Intell. Lab, Volume 148, p. 115-127.

Jackson, J. E., 1991. A User's Guide to Principal Components. s.1.:John Wiley \& Sons, Inc.

Jaffel, I., Taouali, O., Harkat, M. F. \& Messaoud, H., 2016. Fault detection and isolation in nonlinear systems with partial reduced kernel principal component analysis method. T.I. Meas. Control, 40(4), pp. 1289-1296. Jia, F., Martin, E. B. \& Morris, A. J., 1998. Non-linear principal components analysis for process fault detection. Comput. Chem. Eng., Volume 22, pp. S851-S854. 
Korkmaz, S., Goksuluk, D. \& Zararsiz, G., 2014. MVN: An R package for assessing multivariate normality. $R$ J., Volume 6, pp. 151-162.

Kouadri, A., Namoun, A., Zelmat, M. \& Aitouche, M. A., 2013. A statistical-based approach for fault detection in a three tank system. Int. J. Syst. Sci., 44(10), pp. 1783-1792.

Kullback, S. \& Leibler, R., 1951. On information and sufficiency. Ann. Math. Statist., Volume 22, pp. 79-86.

Ku, W., Storer, R. H. \& Georgakis, C., 1995. Disturbance detection and isolation by dynamic principal component analysis. Chemometr. Intell. Lab. Syst., 30(1), pp. 179-196.

Lee, J.-M.et al., 2004. Nonlinear process monitoring using kernel principal component analysis. Chem. Eng. Sci., 59(1), pp. 223-234.

Malinowski, E. R., 1991. Factor Analysis in Chemistry. New York: Wiley Interscience.

Mirin, S. N. S., 2013. Fault detection and monitoring using multiscale PCA. s.1., IEEE 4th Contral and System Graduate Research Colloquium.

Qin, S. \& Dunia, R., 2000. Determining the number of principal components for best reconstruction. J. Process Control, Volume 10, pp. 245-250.

Ramzan, S., Zahid, F. \& Ramzan, S., 2013. Evaluating multivariate normality: a graphical approach. Middle East J. Sci. Res., Volume 13, pp. 254-263.

Rato, T. \& Reis, M., 2013. Fault detection in the Tennessee Eastman banchmark process using Dynamic Principal Components Analysis based on Decorrelated Residuals (DPCA-DR). Chemometr. Intell. Lab., Volume 125, pp. 101-108.

Russell, E., Chiang, L. \& Braatz, D., 2000. Data-driven Methods for Fault Detection and Diagnosis in Chemical Processes. Springer ed. London: s.n.

Sahu, A., Apley, D. W. \& Rungera, G. C., 2014. Feature selection for noisy variation patterns using kernel principal component analysis. Knowledge-Based Syst., Volume 72, pp. 37-47. 
Valle, S., Li, W. \& Qin, S. J., 1999. Selection of the number of principal components: the variance of the reconstruction error criterion with a Comparison to Other Methods. Ind. Eng. Chem. Res., Volume 38, pp. 4389-4401.

Venkatasubramanian, V., Rengaswamy, R., Kavuric, S. N. \& Yind, K., 2013. A review of process fault detection and diagnosis Part III: Process history based methods. Comput. Chem. Eng., Volume 27, pp. 327346.

Wade, M. J., Sánchez, A. \& Katebi, M. R., 2005. On real-time control and process monitoring of wastewater treatment plants: real-time process monitoring. T.I. Meas. Control, 27(3), pp. 173-193.

Wang, B., Jiang, Q. \& Yan, X., 2014. Fault detection and identification using a Kullback-Leibler divergence based multi-block principal component analysis and Bayesian inference. Korean J. Chem. Eng., Volume 31, pp. $930-943$.

Wang, L., Niu, Q. \& Fei, M., 2008. A novel quantum ant colony optimization algorithm and its application to fault diagnosis. T.I. Meas. Control, 30(3-4), pp. 313-329.

Wang, S. \& Chen, Y., 2004. Sensor validation and reconstruction for building central chilling systems based on principal component analysis. Energy Convers. Manage., Volume 45, pp. 673-695.

Wang, Z., 2012. Nonlinear adaptive observer-based fault diagnosis for satellite actuator. Appl. Mech. Mater., Volume 214, pp. 851-855.

Xia, L., Chu, J. \& Geng, Z., 2013. Process monitoring based on improved recursive PCA methods by adaptive extracting principal components. T.I. Meas. Control, 35(8), pp. 1024-1045.

Xiong, L., Liang, J. \& Qian, J., 2007. Multivariate statistical process monitoring of an industrial polypropylene catalyzer reactor with component analysis and kernel density estimation. Chinese J. Chem. Eng., 15(4), pp. 524-532.

Youssef, A., Delpha, C. \& Diallo, D., 2016. An optimal fault detection threshold for early detection using Kullback-Leibler Divergence for unknown distribution data. Signal Process., Volume 120, pp. 266-279. 
Yu, J., 2016. Process monitoring through manifold regularization-based GMM with global/local information. J. Process Control, Volume 45, pp. 84-99.

Zhang, K. et al., 2017a. Assessment of $\mathrm{T}^{2}$ and Q-statistics for detecting additive and multiplicative faults in multivariate statistical process monitoring. J. Franklin Inst., Volume 354, pp. 668-688.

Zhang, K., Dong, J. \& Peng, K., 2017b. A novel dynamic non-Gaussian approach for quality-related fault diagnosis with application to the hot strip mill process. J. Franklin Inst., Volume 354, pp. 702-721.

Zhang, Y., 2009. Enhanced statistical analysis of nonlinear processes using KPCA, KICA and SVM. Chem. Eng. Sci., Volume 64, pp. 801-811.

Zhongda, T., Shujiang, L., Yanhong, W. \& Xiangdong, W., 2016. A multi-model fusion soft sensor modelling method and its application in rotary kiln calcination zone temperature prediction. T.I. Meas. Control, 38(1), pp. 110-124.

Zhuang, G., Li, Y. \& Li, Z., 2016. Fault detection for a class of uncertain nonlinear Markovian jump stochastic systems with mode-dependent time delays and sensor saturation. Int. J. Syst. Sci., 47(7), pp. 1514-1532.

Zhuang, G., Li, Y. \& Lu, J., 2014. Fuzzy fault-detection filtering for uncertain stochastic time-delay systems with randomly missing data. T.I. Meas. Control, 37(2), pp. 242-264.

Zwick, W. R. \& Velicer, W. F., 1989. Comparison of five rules for determining the number of components to retain. Psychol. Bull., 99(3), pp. 432-442. 